\title{
Oguchi disease: suggestion of linkage to markers on chromosome $2 \mathrm{q}$
}

\author{
Marion A Maw, Sheila John, Sibylle Jablonka, Bertram Müller, \\ Govindasamy Kumaramanickavel, Ralph Oehlmann, Michael J Denton, Andreas Gal
}

\begin{abstract}
Oguchi disease is a rare autosomal recessive form of congenital stationary night blindness. The condition is associated with fundus discolouration and abnormally slow dark adaptation. Earlier studies suggested that the $48 \mathrm{kD}$ protein $S$ antigen may be involved in the recovery phase of light transduction. Previous cytogenetic and linkage studies have localised the $S$ antigen gene (SAG) to chromosome $2 q 37.1$. In the present study markers which map to distal chromosome $2 q$ were typed in an inbred Oguchi pedigree. The segregation data obtained suggested that the affected subjects are homozygous by descent for a region between D2S172 and D2S345. An intragenic SAG polymorphism was homozygous in all affected people and a recombination event suggested that SAG maps proximal to D2S345. Collectively, these findings support the hypothesis that a defect in $S$ antigen may be responsible for Oguchi disease.
\end{abstract}

( $(\mathcal{M}$ Med Genet 1995;32:396-398)

Biochemistry

Department

University of Otago, PO Box 56, Dunedin, New Zealand

M A Maw

G Kumaramanickavel

M J Denton

Medical Research

Foundation,

18 College Road,

Madras-600 006,

India

S John

Institut für

Humangenetik,

Medizinische

Universität,

Ratzeburger Allee 160 , D-23538 Lübeck,

Germany

$S$ Jablonka

R Oehlmann

A Gal

Abteiling für

Kinderpoliklinik der

Universität,

Goethestrasse 29

80336 München,

Germany

B Müller

Correspondence to:

Dr Gal

Received 30 August 1994 Revised version accepted for publication

9 December 1994

During normal dark adaptation the sensitivity of the visual system slowly increases, with the rod system becoming considerably more sensitive than the cone system. Dark adaptation of the rods and cones is usually complete within, respectively, 10 and 30 minutes of introduction to a darkened environment. In Oguchi disease, a rare autosomal recessive condition, dark adaptation of the cones appears to proceed normally whereas rod thresholds may take several hours to descend to normal levels. ${ }^{1-3}$ Investigation of the underlying molecular pathology of Oguchi disease may provide insights into both night blindness and the mechanisms which normally control rod dark adaptation.

A reasonable candidate gene for Oguchi disease is that encoding $S$ antigen, also known as arrestin. This gene encodes a $48 \mathrm{kD}$ protein which may be involved in the recovery phase of light transduction. ${ }^{45}$ It has been suggested that $S$ antigen binds to and thereby quenches partially phosphorylated activated rhodopsin $\left(\mathrm{Rh}^{*}\right)$. The binding of $\mathrm{S}$ antigen appeared to stabilise $\mathrm{Rh}^{*}$ and so retard the regeneration of native rhodopsin. Because of the latter finding it was suggested that $S$ antigen serves as a switch that shuts down the rod photoreceptors during prolonged periods of bright illumination. The gene encoding $S$ antigen has been mapped by in situ hybridisation analysis to pädiatrische Genetik,

chromosome $2 \mathrm{q} 37.1^{6}$ and by linkage analysis between D2S172 and D2S125. ${ }^{7}$ Here we describe cosegregation of the Oguchi disease phenotype with polymorphic DNA markers which map to distal chromosome 2q.

\section{Materials and methods}

The pedigree of the family studied here is shown in the figure. The three affected daughters all had night blindness since childhood but retained good visual acuity (6/6). When Humphrey visual field testing was performed, two of the daughters had peripheral field constriction. All three daughters had an abnormal colouration of the fundus which disappeared after 30 to 45 minutes of dark adaptation and extinguished scotopic (rod) and subnormal photopic (cone) electroretinogram (ERG). There was no evidence of the pigmentation associated with retinitis pigmentosa. These clinical findings were consistent with type I Oguchi disease; in type II Oguchi disease the fundus discolouration does not revert to normal in darkness. Both the parents and the unaffected son had normal visual acuity, normal fundus colouration, no history of night blindness, and a photopic ERG within normal limits. parents but was clearly subnormal in the son.

DNA samples from the parents and children were PCR amplified using primers from the MAPPAIRS set (Research Genetics). In each case, the forward primer was radioactively labelled before amplification. The PCR products were then electrophoresed on $6 \%$ denaturing polyacrylamide gels and the gels fixed, dried, and autoradiographed.

A previously described polymorphism at codon 403 of the $S$ antigen gene (GCT (Ala) or ATT (Ile) $)^{8}$ was detected by amplifying DNA samples using flanking primers (forward $5^{\prime}$ GGAGTTTGCTCGCCATAATCTGAA-3' and reverse 5'-CCAGGGGAGAACAAACAAGCTTTA-3'). The 223 bp PCR products were then digested with $H g a \mathrm{I}$, a restriction enzyme which recognises the sequence GACGC and will cleave the PCR product if the codon 403 sequence is GCT but not if it is ATT.

Two point linkage analyses were carried out using the FASTLINK implementation of MLINK. For multipoint analysis, the following map of chromosome $2 \mathrm{q}$ was used: 2 cenD2S126-(16 cM)-D2S172-(4 cM)-SAG-(8 cM)D2S427-(5 cM)-D2S345-(1cM)-D2S338(13 cM)-D2S140-(5 cM)-D2S125-2qter. ${ }^{9}$ Owing to the computational complexity of the 
A

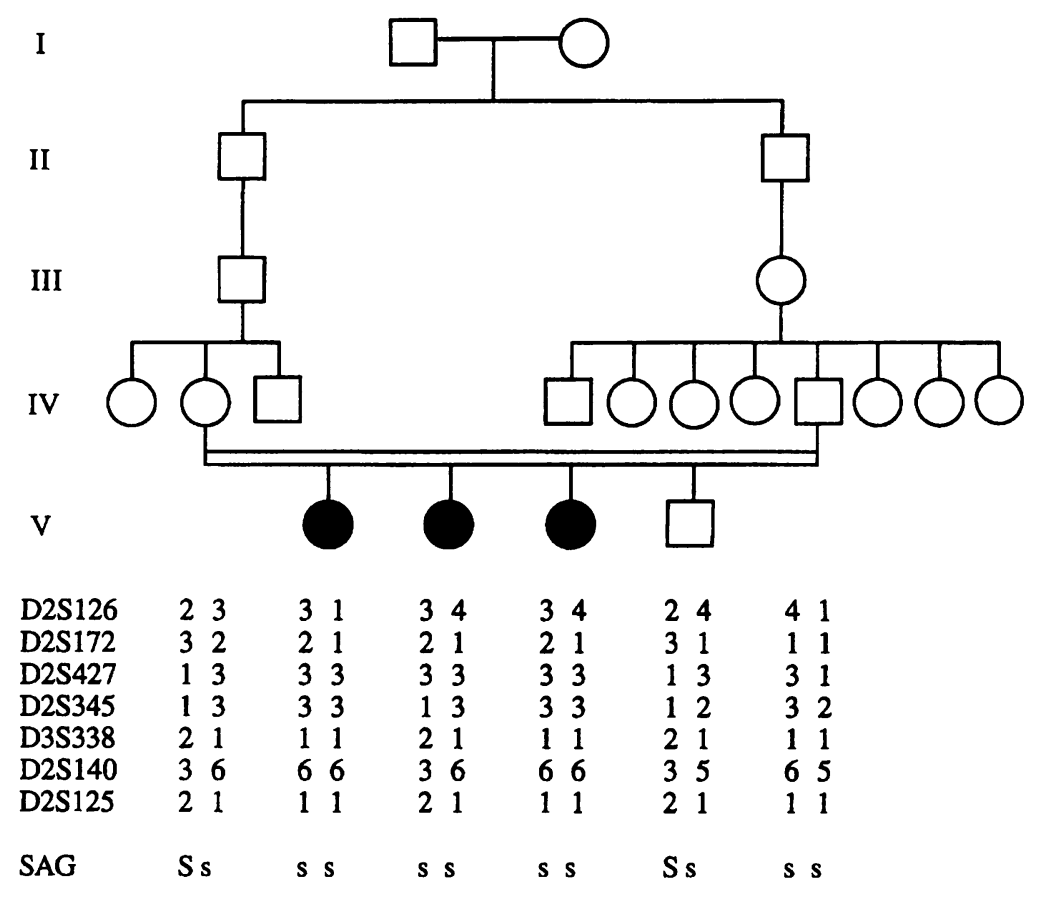

B

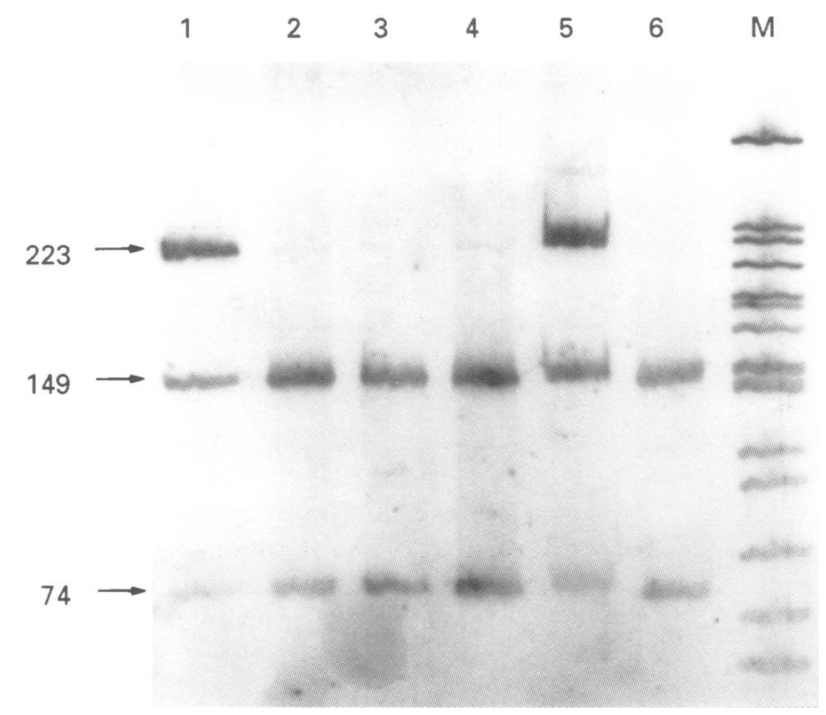

Genetic mapping of the locus for Oguchi disease. (A) Pedigree of the Indian family with Oguchi disease and segregation of chromosome $2 q$ polymorphic markers in that family. Oguchi disease and segregation of chromosome $2 q$ polymorphic markers in that family. $1-6$ denote alleles detected for the D2S126, D2S172, D2S427, D2S345, D2S338,
$D 2 S 140$, and D2S125 microsatellite polymorphisms; $S$ and s denote, respectively, the $A T T$ and GCT sequence polymorphisms at codon 403 in the $S$ antigen gene (SAG). The order and genetic distance of marker loci tested are given in Materials and methods. SAG most likely maps between D2S172 and D2S345. (B) Detection of the SAG codon 403 polymorphism by Hgal digestion. Lane 1, mother; lanes 2-4, affected daughters; lane 5, unaffected son; lane 6 , father; lane $M$, molecular weight marker.

pedigree, we had to resort to overlapping three point analyses using the FASTMAP implementation of LINKMAP. ${ }^{10}$

\section{Results}

In this study microsatellite markers were tested for linkage to the Oguchi disease locus in an inbred pedigree from India. The pedigree involved marriage between third cousins and contained three affected female sibs and one unaffected male sib (figure A). DNA samples from the parents and children were typed for seven microsatellite markers from chromosome $2 \mathrm{q}$ and an intragenic SAG polymorphism (figure A, B). The alleles at SAG, D2S427, D2S345, D2S338, D2S140, and D2S125 formed a haplotype $(\mathrm{s}, 3,3,1,6,1)$ which was carried by both parents and was homozygous both in the first and third affected daughters. The second affected daughter inherited the paternal copy of the same haplotype together with a recombinant maternal haplotype $(s, 3,1,2,3,2)$ such that only D2S427 and SAG were homozygous. The clinically unaffected son was heterozygous for all of the tested markers; he inherited a completely different maternal haplotype $(S, 1,1,2,3,2)$ and a recombinant paternal haplotype (s,3,2,1,5, 1). Two point linkage analysis was performed. If it was assumed that the two alleles at D2S427 segregating in the nuclear family each had an allele frequency of 0.1 and that the frequency of the Oguchi disease gene was 0.002 , then a maximum lod score of 2.19 at a recombination fraction of $\theta=0.0$ was obtained. These assumptions seem reasonable because the marker used was relatively informative in the Indian population (unpublished data) and Oguchi disease is very rare.

Using the loci D2S427 and SAG, multipoint analysis yielded a maximum multipoint lod score of 2.65 for a location of the disease locus right on the top of D2S427. The lod score for a location of the Oguchi locus directly at the SAG locus was $2 \cdot 45$, which was not significantly lower (data not shown).

\section{Discussion}

In the current study, cosegregation of distal chromosome 2q markers with Oguchi disease was shown in an inbred Indian family. The three affected children inherited the same allele combination at D2S172, D2S427, and SAG whereas a different allele combination was inherited by the unaffected child. The six markers SAG, D2S427, D2S345, D2S338, D2S140, and D2S125 formed a haplotype which was carried by both parents and was homozygous in two of the affected children. The remaining affected child inherited a recombinant maternal chromosome such that only D2S427 and SAG were homozygous. These homozygosity mapping data suggest that the Oguchi disease locus in this family is located between D2S172 and D2S345. Furthermore, the alleles observed in the affected child with the recombinant chromosome indicated that SAG also maps proximal to D2S345. A similar assignment of SAG has been defined very recently by multipoint linkage analysis in eight reference families. ${ }^{7}$ Collectively, these data are in line with our original hypothesis that a defect in $S$ antigen may be responsible for Oguchi disease in this family. In this case the parents were both heterozygous for a mutation inherited from one of the people in generation I, that is, the affected 
offspring are likely to be homozygous by descent for the mutation. The typing data were consistent with this scenario.

The family analysed in this study is theoretically large enough to produce a lod score of 3.00 , the minimum value required for statistically significant evidence of linkage. To reach this value, the affected subjects must be homozygous by descent for a sequence variation inherited from a common ancestor, and the meioses occurring in generations I to III of the pedigree must be used fully in calculating the lod score. Oguchi disease is so uncommon that it is highly probable that the affected daughters in this pedigree were homozygous by descent. However, a lod score of 3.00 was not achieved on two point analysis of D2S427 because the assumed allele frequency was too high to provide strong evidence that the marker allele was also homozygous by descent. Nevertheless the observation that both parents carried the same haplotype at SAG, D2S427, D2S345, D2S338, D2S140, and D2S125 suggests that homozygosity by descent may in fact have occurred. The segregation data presented here are collectively suggestive of linkage and it would be of interest to test additional pedigrees with Oguchi disease for linkage to markers from distal chromosome 2q. Because mutations in other genes in the phototransduction pathway have been implicated in both night blindness and retinal degenerations, we are also testing autosomal recessive retinitis pigmentosa pedigrees for linkage to $S$ antigen.

We are grateful for the financial support which the British and German RP Societies have provided for this work since its inception. Funds have also been provided by the Deutsche Forschungsgemeinschaft (DFG, Germany), the National Retinitis Pigmentosa Foundation, Inc (USA), and the New Zealand Health Research Council.

1 Francois J, Verriest G, De Rouck A. La Maladie d'Oguchi. Ophthalmologica 1956;131:1-40.

2 Carr RE, Gouras P. Oguchi's disease. Arch Ophthalmol 1965; 73:646-56.

3 Carr RE, Ripps H. Rhodopsin kinetics and rod adaptation in Oguchi's disease. Invest Ophthalmol Vis Sci 1967;6: 426-36.

4 Palczewski K, Rispoli G, Detwiler PB. The influence of arrestin (48K protein) and rhodopsin kinase on visual transduction. Neumn 1992;8:117-26.

5 Palczewski K, McDowell JH, Takes S, Ingebritsen TS Hargrave PA. Regulation of rhodopsin dephosphorylation by arrestin. $\mathcal{f}$ Biol Chem 1989;264:15770-3.

6 Lu-Kuo J, Ward DC, Spritz RA. Fluorescence in situ hybridization mapping of 25 markers on distal human chromosome $2 \mathrm{q}$ surrounding the human Waardenburg syndrome type I (WS1) locus (PAX3 gene). Genomics 1993;16:173-9.

7 Valverde $\mathrm{D}$, Bayès $\mathrm{M}$, Martínez $\mathrm{I}$, et al. Genetic fine localization of the arrestin (S-antigen) gene $4 \mathrm{cM}$ distal from D2S172. Hum Genet 1994;94:193-4.

8 Sheffield VC, Beck JS, Nichols B, et al. Detection of multiallelic polymorphisms within gene sequences by GC allelic polymorphisms within gene sequences by $\mathrm{GC}$ Hum Genet 1992;50:567-75.

9 Gyapay G, Morissette J, Vignal A, et al. The 1993-1994 Genethon human genetic linkage map. Nature Genet 1994; 7:246-339.

10 Schaffer AA, Gupta SK, Shriram K, Cottingham RW Jr Avoiding recomputation in genetic linkage analysis. Hum Hered 1994;44:225-37. 